
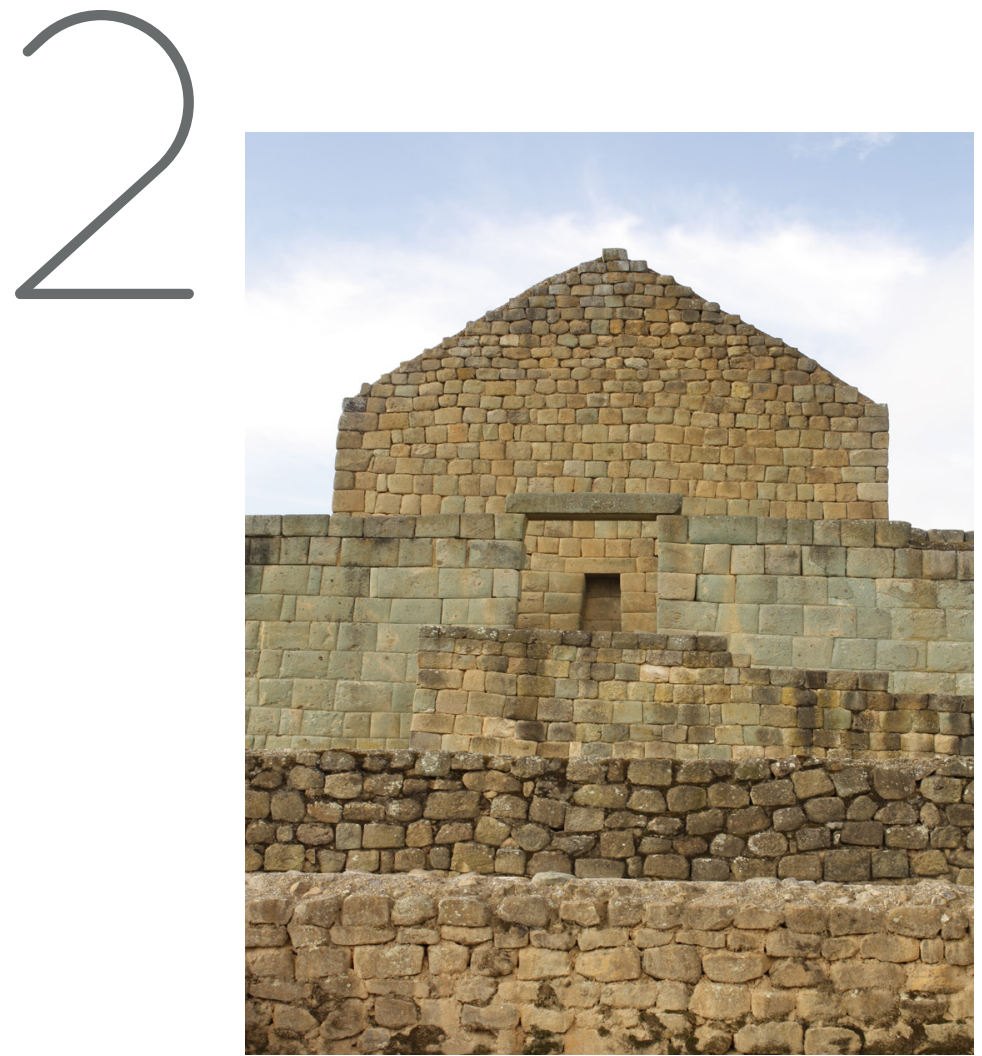

\title{
EN TORNO AL PATRIMONIO CULTURAL Y SU GESTIÓN
}

\author{
Jaramillo Paredes, Diego \\ Profesor de la Universidad del Azuay y de la \\ Universidad de Cuenca.
}

Correspondencia: djaramillo@uazuay.edu.ec 


\title{
Resumen
}

Durante mucho tiempo se consideró al patrimonio cultural únicamente como un campo asociado con las construcciones, y por tanto, directamente asociado con la arquitectura; sin embargo el patrimonio de los pueblos es mucho más que eso, y no solamente que se debe desarquitecturizar la noción del patrimonio, sino que también se debe incluir, necesariamente, la participación y la visión de los miembros de la comunidad, que son, realmente, los constructores y usuarios naturales de los valores patrimoniales.

Este acto de desarquitecturizar el patrimonio abre nuevas y muy amplias perspectivas para tratar este tema.

Palabras clave: Patrimonio, desarquitecturizar el patrimonio, participación de los sujetos-actores en el patrimonio, gestión del patrimonio.

\section{CONCERNING THE CULTURAL HERITAGE AND ITS MANAGEMENT}

\begin{abstract}
For a long time it was considered the cultural heritage solely as a field associated with the buildings, and therefore directly associated with architecture; but the heritage of the people is much more than that, and not only it as to change the Architectural the notion of heritage, but also we must necessarily include participation and vision of community members who really are, the builders and users of natural heritage values.
\end{abstract}

This act to change the Architectural the notion of heritage, open a new and very widespread of new perspectives to address this issue.

Keywords: Heritage, change the Architectural the notion heritage, participation of subjects-actors in heritage, management of heritage. 
El presente artículo pretende contribuir a la reflexión y el debate sobre la conservación del patrimonio cultural y su gestión, a partir de un enfoque orientado a desarquitecturizar el patrimonio cultural, es decir sacarlo de los límites de la disciplina de la arquitectura; para, entenderlo como un campo multidisciplinario y con la participación de múltiples actores.

Desde las visiones y prácticas de la conservación del patrimonio cultural a manos de expertos (producto del iluminismo y del romanticismo), basadas en el reconocimiento exclusivo de valores históricos y artísticos y en las características materiales del bien, hasta la concepción del patrimonio como recurso para el desarrollo integral de la comunidad, ha pasado mucho tiempo y se ha acumulado mucha experiencia teórica y práctica, a través de la cual se ha ido construyendo, cada vez con más fuerza y convencimiento, la visión de que la conservación del patrimonio cultural es una tarea colectiva que incluye una compleja red de involucrados.

Desde la propia definición de patrimonio cultural se puede comprender la participación de los sujetos-actores en su constitución. Participación que nace desde la propia noción de patrimonio y que debería mantenerse de manera permanente durante todo el proceso de su conservación y gestión.

\section{CONCEPTOS BÁSICOS}

El patrimonio cultural es una construcción social, es un sentido de apropiación, de pertenencia, que se construye en la relación social entre sujetos y objetos. Cuando en 
esa relación, la comunidad o parte de ella siente un objeto o bien material o intangible como suyo, aparece el sentido de patrimonio, de bien patrimonial.

"El patrimonio no está limitado al objeto, está en la mente de los ciudadanos que reconocen valores en él. Está en la mente del ciudadano, donde comienza la batalla para la conservación de su herencia cultural" (CARABALLO. 2011. 33)

Bajo esta consideración el patrimonio cultural no es absoluto (igual para toda la comunidad e invariable en el tiempo) sino, por el contrario, es relativo, cambiante en el tiempo y en el espacio.

De otra parte, el concepto de valor patrimonial está también indisolublemente ligado a la presencia y participación de los sujetos sociales, de la comunidad.

Entendemosal valor como un conjunto de características positivas o cualidades percibidas en objetos culturales o sitios por ciertos individuos o grupos; y, como el producto de la interacción entre un bien y su contexto; no emanan del artefacto en sí. (Ver, Mason, 2002)

Los valores son formados en el nexo entre ideas (valor extrínseco) y cosas (valor intrínseco), y por lo tanto los valores son fundamentalmente contingentes; es decir, son construidos socialmente en un espacio y en un tiempo determinado.

"los valores patrimoniales, como parte de un complejo mayor de sistemas de valores sociales, son conceptos éticos, socialmente cambiantes, aceptados y deseados 
como ideales en un determinado contexto social e histórico... Los valores no existen fuera de las relaciones sociales establecidas, ni son necesariamente comunes a todas ellas. El valor es un concepto que por un lado expresa las necesidades cambiantes del hombre, y por otro fija la significación positiva de los fenómenos naturales y sociales para la existencia y desarrollo de la comunidad" (CARABALLO, 2011.26)

"Los valores no pueden considerarse homogéneos ni permanentes ni socialmente objetivos. Estos valores abstractos son reflejados y trasladados a bienes $y$ manifestaciones culturales que sirven de referente a las aspiraciones y discursos identitarios colectivos. Es así que el objeto o la manifestación estará cargado de múltiples valores, que hacen de él un símbolo de identidad multi-discursivo" (CARABALLO, 2011.27)

Los valores patrimoniales pueden ser reconocidos de diversa manera por los integrantes de una comunidad, a través de los llamados atributos, pudiendo reconocerse en un mismo atributo diversos valores que pueden ser, incluso, contradictorios entre sí, lo que demuestra el grado de subjetividad de los valores patrimoniales.

Resumiendo diríamos que, conceptualmente, no hay patrimonio sin actores, sin sujetos, sin comunidad.

Ahora bien, luego de lo señalado, nos vamos a orientar a la gestión del patrimonio y enfocarnos en la conservación basada en valores, para enfatizar, que ésta es una tarea multidisciplinaria y con la participación de múltiples actores. 


\section{LA CONSERVACIÓN BASADA EN VALORES}

La pregunta que siempre está subyacente en las intervenciones que se realizan en el patrimonio cultural edificado es: ¿los valores que los profesionales pretenden preservar en un sitio son efectivamente los valores que reciben audiencia en el público general? La respuesta, bien sabemos, en la gran mayoría de casos es negativa. Frente a esta situación y contrariamente al enfoque tradicional de la conservación que prioriza las cualidades de la materialidad de los bienes patrimoniales, cada vez se impone la visión de la conservación basada en valores que propone que la conservación del patrimonio no es una tarea técnica-científica de una determinada disciplina, sino una tarea multidisciplinaria de interacción con la comunidad.

Las decisiones sobre el patrimonio deben ser tomadas en base a responder, entre otras, una pregunta fundamental: ¿qué conservar?

La conservación basada en valores se define por algunos aspectos fundamentales:

a. Por otorgarle un papel central a la significancia en la toma de decisiones. Por significancia cultural se puede entender como la importancia de un sitio determinada por el conjunto de valores atribuidos a ese sitio. Los valores considerados deben incluir los identificados por expertos -historiadores de arte, arqueólogos, arquitectos y por nuevos actores.

b. Se opone a una visión desde las cualidades de la materialidad y fija la prioridad sobre el entendimiento de por qué la materialidad es valiosa. 
c. Para este enfoque de la preservación basada en valores, es crucial el ejercicio de identificar e investigar los valores que aportan a la significancia cultural del sitio.

Muchos son los argumentos que apoyan la conservación basada en valores, entre los más destacados podemos mencionar:

- Permite un entendimiento holístico de los sitios; es decir su significancia cultural más allá de la pura materialidad.

- Lleva a un reconocimiento y la inclusión de un rango más amplio de actores, no sólo de diversas disciplinas, sino de actores de la comunidad que participan no desde ninguna experticia profesional, sino desde su condición de ciudadanos.

- Se basa en un conocimiento comprensivo de los valores del sitio, el cual es esencial para dar soporte a una visión a largo plazo de su cuidado.

Siguiendo a Jokiletho (2006), podemos apuntar algunos aspectos importantes de este enfoque de la conservación basada en valores:

- Los valores y la significancia solamente pueden construirse en comunicación y diálogo con otros miembros de la sociedad, formando una identidad cultural para la comunidad. Como ya señalamos líneas arriba, el valor es un constructo cultural, una construcción dialógica.

- Reconociendo que la generación de valores y sentidos requiere de un diálogo, los problemas pueden ser interpretados como la pérdida de horizontes comunes o valores compartidos, lo cual va más allá de la individualidad personal y destaca la responsabilidad compartida. 
- Todas las creencias son válidas de manera igual y por lo tanto la verdad es relativa a su situación, el contexto y los individuos relacionados; lo que, consecuentemente, nos pone frente a la necesidad de relativizar las intervenciones en el patrimonio, de acuerdo a los casos particulares.

Ahora bien, los valores patrimoniales que tradicionalmente han sido priorizados o visibilizados, han sido los artísticos y los históricos. Una mirada sobre los inventarios patrimoniales a nivel nacional o internacional, evidencia lo señalado.

Sin embargo, importantes intentos se realizan para ampliar la gama de los valores patrimoniales; intentos que también han sido parte de las preocupaciones de los propios organismos internacionales involucrados en el tema.

Tipologías de valores patrimoniales planteados por diversos eruditos y organismos

\begin{tabular}{|l|l|l|l|l|}
\hline \multicolumn{1}{|c|}{ Riegl 1902 } & Lipe 1984 & $\begin{array}{c}\text { Carta de } \\
\text { Burra } \\
1988\end{array}$ & Frey 1997 & $\begin{array}{l}\text { Patrimonio } \\
\text { Inglés 1997 }\end{array}$ \\
\hline Edad & Económicos & Estético & Monetario & Cultural \\
\hline Históricos & Estéticos & Histórico & Opción & Educacional \\
\hline Conmemorativos & Asociativos & Científico & Existencia & Recurso \\
\hline Usos & Simbólicos & Social & Legado & Recreacional \\
\hline Nuevos & Internacional & & Prestigio & Estético \\
\hline & & & Educacional & \\
\hline
\end{tabular}

Alois Riegl (Austria, 1858, - 1905) fue un historiador del arte austrohúngaro, uno de los principales impulsores del 
formalismo. Fue uno de los fundadores de la crítica de arte como disciplina autónoma.

Dr. William D. Lipe. Ph.D. Arqueólogo con experticia en la región de Norte de América, métodos y teoría de la arqueología y el manejo de recursos culturales.

Dr. Bruno Frey. (Suiza 1941) Economista. Quizá su investigación más famosa se relaciona con la crítica del Homo. Otro campo de sus contribuciones que ha ganado una atención considerable es su análisis económico de la felicidad. En algunas publicaciones analiza la "economía del arte", usando un punto de vista económico para analizar fenómenos relacionados con las actividades artísticas.

\begin{tabular}{|l|l|}
\hline \multicolumn{2}{|c|}{$\begin{array}{c}\text { Tipología provisional de valores patrimoniales (The Getty } \\
\text { Conservation Institute, Los Angeles, 2002) }\end{array}$} \\
\hline Valores socio-culturales & Valores económicos \\
\hline Históricos & Valores de uso \\
\hline Culturales/simbólicos & Valores de no uso \\
\hline Espirituales/religiosos & Existencia \\
\hline Estéticos & Opción \\
\hline & Legado \\
\hline
\end{tabular}

La hipótesis de trabajo detrás de la tipología propuesta es que las categorías abarcan la mayoría de los valores patrimoniales que den forma a las decisiones a tomarse en la planificación y el manejo de la conservación. Las dos categorías mayores, valores socio-culturales y económicos, no se refieren a conjuntos de valores diferentes o discretos, sino consisten en dos vías alternas para entender y categorizar el mismo abanico de valores patrimoniales. 


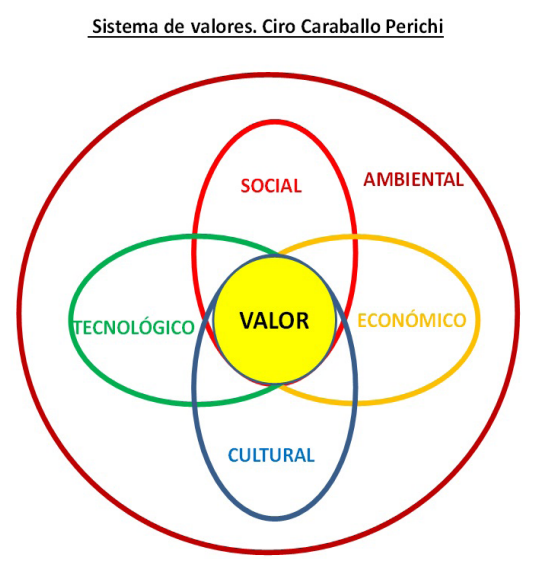

\begin{tabular}{|c|c|c|c|c|}
\hline \multicolumn{5}{|c|}{ MATRIZ DE NARA } \\
\hline & \multicolumn{4}{|c|}{ dimensiones } \\
\hline aspectos & Estética & Histórica & $\begin{array}{l}\text { Científica / } \\
\text { tecnológica }\end{array}$ & Social \\
\hline Forma y diseño & & & & \\
\hline $\begin{array}{l}\text { Materiales y } \\
\text { substancia }\end{array}$ & & & & \\
\hline \multicolumn{5}{|l|}{ Uso y función } \\
\hline \multicolumn{5}{|l|}{$\begin{array}{l}\text { Tradición, técnicas, } \\
\text { experticias }\end{array}$} \\
\hline \multicolumn{5}{|l|}{$\begin{array}{l}\text { Lugares y } \\
\text { asentamientos }\end{array}$} \\
\hline Espíritu y sentimiento & & & & \\
\hline
\end{tabular}

La llamada Matriz de Nara, surgida de la Carta de Nara sobre la autenticidad del patrimonio cultural, plantea la existencia de valores estéticos, históricos, científicos y sociales y señala que las fuentes de verificación de la información de esos valores se deberían encontrar en uno o más de los denominados aspectos. 
Para Ciro Caraballo la identificación de valores debe ser un ejercicio democrático e incluyente, que dé espacio a múltiples actores, permitiendo, así, el ejercicio de un derecho cultural de toda sociedad. Este concepto se señala en la Carta de Enane (ICOMOS 2005).

Como se puede ver en esta rápida síntesis de algunas propuestas de tipologías de valores, éstos son variados y en muchas ocasiones conflictivos; y, en todos los casos, rebasan las nociones de la arquitectura, de la historia, el arte y la arqueología.

Resumiendo, nos preguntaríamos ¿seguimos, anacrónicamente, conservando el patrimonio a partir de las características materiales de éste, o nos enfocamos en una conservación basada en valores? Si damos el necesario paso a esta segunda opción, el cuestionamiento fundamental para la gestión del patrimonio es ¿los valores de quienes?

Luego de la trayectoria seguida en este artículo, parecería estar claro que no tiene sustento la idea del patrimonio constituido a partir de la exclusiva mirada de los expertos, normalmente arquitectos, arqueólogos, historiadores, sino que hay que abrir el abanico tanto como sea posible para abarcar a los distintos profesionales de las disciplinas involucradas por el espectro de valores, como a la comunidad.

Algunos autores, respondiendo a esta necesidad, plantean un abanico que se puede concretar en:

- La academia y los especialistas, que construyen los discursos sobre el bien y su significancia. 
- El Estado, que establece políticas, normativas y destina recursos.

- La sociedad civil, que dispone de programas e inversiones puntuales y representa a la comunidad inmediata.

- La comunidad que convive con el bien.

Lo que interesa en un proceso de planificación para la conservación del patrimonio, con el objetivo de garantizar su sostenibilidad en el tiempo, es que los valores de todos los involucrados puedan ser expresados y discutidos.

Este proceso complejo de identificación de valores, sin lugar a dudas, requerirá de un conjunto de métodos cuantitativos y cualitativos y herramientas diferentes, aportados desde diversas disciplinas, que tendrán que ajustarse para cada proyecto o caso específico. De todas maneras es indispensable la participación de profesionales de la conservación que comprendan el objeto dentro de un contextoampliadoy, así, contribuyan a consolidarunanoción de conservación como una disciplina interdisciplinaria, técnica y basada en las ciencias humanas.

Luego de lo expuesto, es notoria la poca significación que en el complejo proceso de conservación del patrimonio, puede tener la llamada, por los organismos públicos responsables de la conservación del patrimonio, socialización de los proyectos de intervención o conservación. No se trata de consultarle a la comunidad sobre un proyecto ya definido y encontrar las maneras de su aceptación o aprobación; se trata de que todo el proceso, desde la identificación de los valores patrimoniales del sitio, incluya, como aquí lo hemos expuesto, a la comunidad. Participación que estamos convencidos debe darse porque 
nuestra concepción del patrimonio cultural así lo exige; porque la gestión contemporánea del patrimonio así lo requiere y porque a través de lograr esa participación de la comunidad, los organismos de gobierno están cumpliendo una tarea fundamental de su razón de ser: la construcción de ciudadanía; tema éste para otro artículo.

\section{BIBLIOGRAFÍA}

- CARABAllo, C. 2011, Patrimonio Cultural, un enfoque diverso y comprometido, UNESCO, México, 11-71.

- Carta de Nara. 1994, ICOMOS

- MASON, R. 2006. Theoretical and Practical Arguments for Values-Centered Preservation. Cultural Resource Management: The Journal of Heritage Stewardship, 3, 21-48.

- MASON, R. 2002, Assessing Values in Conservation Planning: Methodological Issues and Choices, en Assessing the Values of Cultural Heritage, Marta de la Torre, Editora, The getty Conservation Institute, Los Angeles, 5-30.

- JOKILEHTO, J. 2006, Considerations on authenticity and integrity in world heritage contex. City \& Time 2 (1): 1.(online) URL: http//www.ct.ceci-br.org, 1-16

- $\quad$ STOVEL, H. 2007, Effective use of authenticity and integrity as world heritage qualiifying conditions. City \& Time 2 (3): 3. [online] URL: http://www.ct.ceci-br.org, 21-36 
